\title{
On the Blaschke trihedrons of a line congruence
}

\author{
Sadullah Celik and Emin Ozyilmaz \\ Department of Mathematics, Faculty of Science, University of Ege, Izmir, Turkey \\ Received: 20 October 2015, Revised: 21 October 2015, Accepted: 6 December 2015 \\ Published online: 30 January 2016.
}

\begin{abstract}
In this study, it is aimed to find relation between the Blaschke vectors of parameter ruled surfaces of a line congruence which are not principle ruled surfaces. By this relation, we can find some basic formulae of the line space. (e.g.the Mannheim's and Liouville's formulae.
\end{abstract}

Keywords: Dual Space, Blaschke Trihedron, Dual Curvature.

\section{Introduction}

A set of one-parameter of lines is called a ruled surface. Ruled surfaces, especially, developable ruled surfaces are used and applied several areas in mathematics and engineering,[1].

Dual number is a useful tool for line trajectories. Indeed, lines in Euclidean 3-space can be expressed by unit dual vectors and there is one to one correspondence between points of dual unit sphere $S^{2}$ and lines of Euclidean 3-space due to Study theorem. For detail, see $[6,7,8,9]$.

On the other hand, if we take two parameters in unit dual vectors, we have a line congruence. In practices, the line congruence defines a family of ruled surfaces. The study of line congruence was started by E.Kummer [5] ,in which he gave a classificasion of those of order one. The applications of the line geometry and dual number representations of line trajectories have been developed by Blaschke [4], and Muller [10].

In [2] and [3], Caliskan gave a formulae between the Blaschke vectors of any ruled surface $\overrightarrow{R_{1}}$ and the parameter ruled surfaces $\overrightarrow{R_{11}}, \overrightarrow{R_{21}}$. Here, he used the parameter ruled surfaces by choosing as principle ruled surfaces.

In this study, it is aimed to find relation between the Blaschke vectors of parameter ruled surfaces of a line congruence which are not principle ruled surfaces. By this relation, we can find some basic formulae of the line space. (e.g.the Manheim's and Liouville's formulae).

\section{Preliminaries}

Let $\mathrm{A}=\mathrm{a}+\varepsilon a_{0}$ be a dual number, $A \in I D=\left\{\left(a, a_{0}\right) \mid a, a_{0} \in I R\right\}$ and $I D$ be a commutative ring with a unit element. We call dual number $\varepsilon=(0,1) \in I D$ as dual unit which satisfies $\varepsilon^{2}=(0,0) .\left(D^{3},+\right)$ is a module on the dual number ring. We call 
it $I D$-module, and dual vectors are the elements of this modul. We denote a unit vector $\vec{A}$ as

$$
\vec{A}=\left(\vec{a}, \overrightarrow{a_{0}}\right)=\vec{a}+\varepsilon \overrightarrow{a_{0}},<\vec{a}, \vec{a}>=1,<\vec{a}, \vec{a}_{0}>=0
$$

where $\vec{a}, \overrightarrow{a_{0}} \in R^{3}$.

Definition 1. The scalar product of two dual vectors $\vec{A}=\vec{a}+\varepsilon \overrightarrow{a_{0}}$ and $\vec{B}=\vec{b}+\varepsilon \overrightarrow{b_{0}}$ is given by

$$
<\vec{A}, \vec{B}>=<\vec{a}, \vec{b}>+\varepsilon\left(<\vec{a}, \overrightarrow{b_{0}}>+<\overrightarrow{a_{0}}, \vec{b}>\right)
$$

Definition 2. [4] The vectoral product between two dual vectors $\vec{A}=\vec{a}+\varepsilon \overrightarrow{a_{0}}$ and $\vec{B}=\vec{b}+\varepsilon \overrightarrow{b_{0}}$ is defined by

$$
\vec{A} \wedge \vec{B}=\vec{a} \wedge \vec{b}+\varepsilon\left(\vec{a} \wedge \overrightarrow{b_{0}}+\overrightarrow{a_{0}} \wedge \vec{b}\right)
$$

\section{The ruled surface and the line congruence}

The Blaschke trihedron $\left\{\overrightarrow{R_{1}}, \overrightarrow{R_{2}}, \overrightarrow{R_{3}}\right\}$ depends on the striction point of the ruled surface $\overrightarrow{R_{1}}(t)$ in dual space $I D^{3}$,[4]. According to this, the first axis $\overrightarrow{R_{1}}(t)$ of the trihedron is the generator which passes from the striction point of the ruled surface, the second axis $\overrightarrow{R_{2}}(t)$ is normal of the surface at this point and finally the third axis $\overrightarrow{R_{3}}(t)$ is the tangent of the striction line at this point. The derivative formulae of the Blaschke trihedron $\left\{\overrightarrow{R_{1}}, \overrightarrow{R_{2}}, \overrightarrow{R_{3}}\right\}$ with respect to dual arc parameter $\mathrm{S}$ of the striction curve are written

$$
\overrightarrow{R_{1}^{\prime}}=P \overrightarrow{R_{2}}, \overrightarrow{R_{2}^{\prime}}=-P \overrightarrow{R_{1}}+Q \overrightarrow{R_{3}}, \overrightarrow{R_{3}^{\prime}}=-Q \overrightarrow{R_{2}}
$$

where $P=\left\|\overrightarrow{R_{1}^{\prime}}\right\|, Q=\operatorname{det} \frac{\left(R_{1}, R_{1}^{\prime}, R_{1}^{\prime \prime}\right)}{P^{2}}$

A ruled surface is given as dual vectorial function by

$$
\overrightarrow{R_{1}}(t)=\vec{r}(t)+\varepsilon \overrightarrow{r_{0}}(t),{\overrightarrow{R_{1}}}^{2}=1
$$

Definition 3. [3] The dual spherical curvature of the ruled surface $\overrightarrow{R_{1}}(t)$ is defined as follows:

$$
\Sigma=\frac{Q}{P}
$$

On the other hand, the line congruence in $I D^{3}$ can be represented by a unit dual vector which depends on two real parameters $\mathrm{u}$ and $\mathrm{v}$ as follows:

$$
\vec{R}(u, v)=\vec{r}(u, v)+\varepsilon \overrightarrow{r_{0}}(u, v), \quad \vec{R}^{2}=1
$$

The dual arc element of a ruled surface of the line congruence can be given as 


$$
d S^{2}=d \vec{R}^{2}=\left(\vec{R}_{u} d u+\vec{R}_{v} d v\right)^{2}=E d u^{2}+2 F d u d v+G d v^{2},
$$

where $E=e+\varepsilon e_{0}=<\vec{R}_{u}, \vec{R}_{u}>, F=f+\varepsilon f_{0}=<\vec{R}_{u}, \vec{R}_{v}>$ and $G=g+\varepsilon g_{0}=<\vec{R}_{v}, \vec{R}_{v}>$. Moreover, the differential form I and II of the line congruence are

$$
\begin{gathered}
I=e d u^{2}+2 f d u d v+g d v^{2} \\
I I=e_{0} d u^{2}+2 f_{0} d u d v+g_{0} d v^{2},
\end{gathered}
$$

respectively. Thus, we have

$$
d S^{2}=I+\varepsilon I I
$$

Definition 4. [4] The drall of a ruled surface of a line congruence can be written as follows:

$$
\frac{1}{d}=\frac{I}{2 I I}
$$

4 The relations among the magnitudes of the ruled surface $\overrightarrow{R_{1}}, \overrightarrow{R_{11}}$ and $\overrightarrow{R_{21}}$

Let us consider a ruled surface $\vec{R}=\overrightarrow{R_{1}}(t)$ of the line congruence $\vec{R}=\vec{R}(u, v)$ where $u$ and $v$ are the functions of $t$. Let us write the parameter ruled surfaces of the line congruence as

$$
\overrightarrow{R_{1} 1}=\overrightarrow{R_{1}} 1\left(u, v_{0}\right), \overrightarrow{R_{1} 1^{2}}=1
$$

and

$$
\overrightarrow{R_{2} 1}=\overrightarrow{R_{2} 1}\left(u_{0}, v\right),{\overrightarrow{R_{2}}}^{2}=1
$$

where the ruled surfaces $\overrightarrow{R_{1}}, \overrightarrow{R_{1}} 1$ and $\overrightarrow{R_{2}} 1$ have common line defined as

$$
\overrightarrow{R_{0}}=\vec{R}\left(u_{0}, v_{0}\right)=\overrightarrow{R_{1} 1}\left(u_{0}, v_{0}\right)=\overrightarrow{R_{2} 1}\left(u_{0}, v_{0}\right)
$$

The Blaschke trihedrons of these ruled surfaces are given by

$$
\left\{\overrightarrow{R_{0}}, \overrightarrow{R_{2}}, \overrightarrow{R_{3}}\right\},\left\{\overrightarrow{R_{0}}, \overrightarrow{R_{1} 2}, \overrightarrow{R_{1} 3}\right\},\left\{\overrightarrow{R_{0}}, \overrightarrow{R_{2} 2}, \overrightarrow{R_{2}} 3\right\}
$$

and hence, one can get

$$
\begin{gathered}
\overrightarrow{R_{1}^{\prime}}=P \overrightarrow{R_{2}}, \overrightarrow{R_{2}^{\prime}}=-P \overrightarrow{R_{1}}+Q \overrightarrow{R_{3}}, \overrightarrow{R_{3}^{\prime}}=-Q \overrightarrow{R_{2}}, \\
{\overrightarrow{R_{1} 1^{\prime}}}^{\prime}=P_{1} \overrightarrow{R_{1} 2}, \overrightarrow{R_{1} 2^{\prime}}=-P_{1} \overrightarrow{R_{1} 1}+Q_{1} \overrightarrow{R_{1} 3}, \overrightarrow{R_{1} 3^{\prime}}=-Q_{1} \overrightarrow{R_{1} 2} \\
{\overrightarrow{R_{2}}}^{\prime}=P_{2} \overrightarrow{R_{2} 2}, \overrightarrow{R_{22}}=-P_{2} \overrightarrow{R_{21}}+Q_{2} \overrightarrow{R_{23}}, \overrightarrow{R_{23}}=-Q_{2} \overrightarrow{R_{22}}
\end{gathered}
$$


where $P_{1}=\sqrt{\overrightarrow{R_{u}^{2}}}=\sqrt{E}, P_{2}=\sqrt{{\overrightarrow{R_{v}^{2}}}^{2}}=\sqrt{G}$, and $Q_{1}, Q_{2}$ are dual curvatures of parameter ruled surfaces. The dual arc elements of these ruled surfaces are $\overrightarrow{R_{1}}, \overrightarrow{R_{11}}$ and $\overrightarrow{R_{21}}$ can be given respectively as

$$
d S=P d t, d S_{1}=P_{1} d u=\sqrt{E} d u, d S_{2}=P_{2} d v=\sqrt{G} d v
$$

Moreover, the Blaschke vectors of the Blaschke trihedrons are given by

$$
\vec{B}=Q \overrightarrow{R_{0}}+P \overrightarrow{R_{3}}, \overrightarrow{B_{1}}=Q_{1} \overrightarrow{R_{0}}+P_{1} \overrightarrow{R_{13}}, \overrightarrow{B_{2}}=Q_{2} \overrightarrow{R_{0}}+P_{2} \overrightarrow{R_{23}}
$$

If we choose the parameter ruled surfaces as principle ruled surfaces, we may write $F=\overrightarrow{R_{u}} \overrightarrow{R_{v}}=0$, [2]. In this study we suppose that $F \neq 0$. Let us consider any parameter ruled surfaces of the line congruence $\vec{R}=\vec{R}(u, v), \overrightarrow{R^{2}}=1$.

Remark. The second edges of the parameter ruled surfaces can be written by

$$
\overrightarrow{R_{12}}=\frac{{\overrightarrow{R_{11}}}^{\prime}}{P_{1}}=\frac{\vec{R}_{u}}{\sqrt{E}}, \overrightarrow{R_{22}}=\frac{{\overrightarrow{R_{21}}}^{\prime}}{P_{2}}=\frac{\vec{R}_{v}}{\sqrt{G}}
$$

From (4.10), we have

$$
\overrightarrow{R_{0}}=\frac{\overrightarrow{R_{u}} \wedge \overrightarrow{R_{v}}}{\left\|\overrightarrow{R_{u}} \wedge \overrightarrow{R_{v}}\right\|}=\frac{\overrightarrow{R_{u}} \wedge \overrightarrow{R_{v}}}{\sqrt{E G} \sin \Theta}
$$

and

$$
\overrightarrow{R_{2}}=\frac{\overrightarrow{R_{1}^{\prime}}}{P}=\sqrt{E} \frac{d u}{d S} \overrightarrow{R_{12}}+\sqrt{G} \frac{d v}{d S} \overrightarrow{R_{22}}
$$

On the other hand, let us consider the dual angle between the edges $\overrightarrow{R_{12}}$ and $\overrightarrow{R_{22}}$ as $\Theta$, and the dual angle between the edges $\overrightarrow{R_{2}}$ and $\overrightarrow{R_{12}}$ as $\Phi$. If we apply dot product both sides (4.12) with $\overrightarrow{R_{12}}$ and $\overrightarrow{R_{22}}$, we have

$$
\begin{gathered}
\overrightarrow{R_{2}} \overrightarrow{R_{12}}=\cos \Phi=\sqrt{E} \frac{d u}{d S}+\cos \Theta \sqrt{G} \frac{d v}{d S}, \\
\overrightarrow{R_{2}} \overrightarrow{R_{22}}=\cos (\Theta-\Phi)=\cos \Theta \sqrt{E} \frac{d u}{d S}+\sqrt{G} \frac{d v}{d S} .
\end{gathered}
$$

Thus, from (4.13) and (4.14)

$$
\begin{gathered}
\frac{\sin (\Theta-\Phi)}{\sin \Theta}=\sqrt{E} \frac{d u}{d S} \\
\frac{\sin \Phi}{\sin \Theta}=\sqrt{G} \frac{d v}{d S}
\end{gathered}
$$

are written. Finally, putting (4.15) and (4.16) into (4.12), we have the following equation between the second edges of the Blaschke trihedrons of the ruled surfaces $\overrightarrow{R_{2}}, \overrightarrow{R_{12}}$ and $\overrightarrow{R_{22}}$ by

$$
\overrightarrow{R_{2}}=\frac{\sin (\Theta-\Phi)}{\sin \Theta} \overrightarrow{R_{12}}+\frac{\sin \Phi}{\sin \Theta} \overrightarrow{R_{22}}
$$


Moreover, by the definition of the angle $\Theta$ between $\overrightarrow{R_{12}}$ and $\overrightarrow{R_{22}}$ we write

$$
\overrightarrow{R_{12}} \overrightarrow{R_{22}}=\cos \Theta
$$

Then, from (4.18) we have

$$
\begin{gathered}
\frac{\sin (\Theta-\Phi)}{\sin \Theta}=\frac{d S_{1}}{d S}=\sqrt{E} \frac{d u}{d S} \\
\frac{\sin \Phi}{\sin \Theta}=\frac{d S_{2}}{d S}=\sqrt{G} \frac{d v}{d S}
\end{gathered}
$$

Corollary 1. The third elements $\overrightarrow{R_{3}}, \overrightarrow{R_{13}}$ and $\overrightarrow{R_{23}}$ of the Blaschke trihedrons of the ruled surfaces $\overrightarrow{R_{1}}, \overrightarrow{R_{11}}$ and $\overrightarrow{R_{21}}$ are lineer dependent as follows:

$$
\overrightarrow{R_{3}}=\frac{\sin (\Theta-\Phi)}{\sin \Theta} \overrightarrow{R_{13}}+\frac{\sin \Phi}{\sin \Theta} \overrightarrow{R_{23}}
$$

Proof. If we substitute the equation (4.17) in $\overrightarrow{R_{3}}=\overrightarrow{R_{0}} \wedge \overrightarrow{R_{2}}$ and consider the Blaschke trihedrons of the parameter ruled surfaces $\overrightarrow{R_{11}}$ and $\overrightarrow{R_{21}}$ we obtain (4.21).

Theorem 1. The third elements $\overrightarrow{R_{13}}$ and $\overrightarrow{R_{23}}$ of the parameter ruled surfaces $\overrightarrow{R_{11}}$ and $\overrightarrow{R_{21}}$ can be expressed by dual vectors $\overrightarrow{R_{12}}$ and $\overrightarrow{R_{21}}$ as follows:

$$
\begin{aligned}
& \overrightarrow{R_{13}}=\frac{1}{\sin \Theta}\left(-\cos \Theta \overrightarrow{R_{12}}+\overrightarrow{R_{22}}\right) \\
& \overrightarrow{R_{23}}=\frac{1}{\sin \Theta}\left(-\overrightarrow{R_{12}}+\cos \Theta \overrightarrow{R_{22}}\right)
\end{aligned}
$$

Proof. From the equations (4.4) and (4.11) we write

$$
\begin{aligned}
& \overrightarrow{R_{12}} \wedge \overrightarrow{R_{13}}=\overrightarrow{R_{0}}, \overrightarrow{R_{22}} \wedge \overrightarrow{R_{23}}=\overrightarrow{R_{0}}, \overrightarrow{R_{12}} \wedge \overrightarrow{R_{22}}=\sin \Theta \overrightarrow{R_{0}} \\
& \overrightarrow{R_{1}} \wedge\left(\overrightarrow{R_{13}}-\frac{\overrightarrow{R_{22}}}{\sin \Theta}\right)=\overrightarrow{0} \Rightarrow \sin \Theta \overrightarrow{R_{13}}-\overrightarrow{R_{2} 2}=M \overrightarrow{R_{12}} \\
& \overrightarrow{R_{22}} \wedge\left(\overrightarrow{R_{23}}+\frac{\overrightarrow{R_{12}}}{\sin \Theta}\right)=\overrightarrow{0} \Rightarrow \sin \Theta \overrightarrow{R_{23}}+\overrightarrow{R_{12}}=N \overrightarrow{R_{22}}
\end{aligned}
$$

Here, $M$ and $N$ are dual scalars. And if we apply dot product of (4.25) and (4.26) by the dual vectors $\overrightarrow{R_{12}}$ and $\overrightarrow{R_{22}}$ respectively, and also with the relation (4.4) and (4.21), we have $M=-\cos \Theta$ and $N=\cos \Theta$. Finally, substuting the dual vectors $M$ and $N$ in (4.25) and (4.26), we obtain (4.22) and (4.23).

Corollary 2. The Blaschke vectors of the ruled surfaces $\overrightarrow{R_{1}}, \overrightarrow{R_{11}}$ and $\overrightarrow{R_{21}}$, which are defined in (4.9), can be written according to unit dual vectors $\overrightarrow{R_{12}}, \overrightarrow{R_{22}}$ and $\overrightarrow{R_{0}}$ as follows:

$$
\vec{B}=Q \overrightarrow{R_{0}}-P\left(\frac{\cos (\Theta-\Phi)}{\sinh \Theta} \overrightarrow{R_{12}}-\frac{\cos \Phi}{\sin \Theta} \overrightarrow{R_{22}}\right)
$$




$$
\begin{aligned}
& \overrightarrow{B_{1}}=Q_{1} \overrightarrow{R_{0}}+\frac{P_{1}}{\sin \Theta}\left(-\cos \Theta \overrightarrow{R_{12}}+\overrightarrow{R_{22}}\right) \\
& \overrightarrow{B_{2}}=Q_{2} \overrightarrow{R_{0}}+\frac{P_{2}}{\sin \Theta}\left(-\overrightarrow{R_{12}}+\cos \Theta \overrightarrow{R_{22}}\right)
\end{aligned}
$$

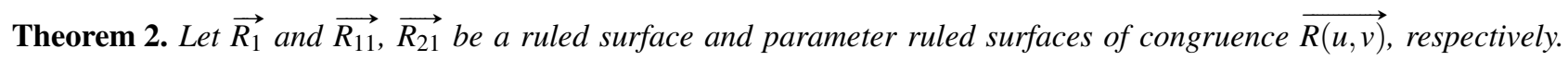
Then, we have

$$
\begin{gathered}
\overrightarrow{R_{12}} \frac{\partial \overrightarrow{R_{22}}}{\partial S_{1}}=-\overrightarrow{R_{22}} \cdot \frac{\partial \overrightarrow{R_{12}}}{\partial S_{1}}=\frac{(\sqrt{E})_{v}-\cos \Theta(\sqrt{G})_{u}}{\sqrt{E G}} \\
\overrightarrow{R_{22}} \frac{\partial \overrightarrow{R_{12}}}{\partial S_{2}}=-\overrightarrow{R_{12}} \cdot \frac{\partial \overrightarrow{R_{22}}}{\partial S_{2}}=\frac{(\sqrt{G})_{u}-\cos \Theta(\sqrt{E})_{v}}{\sqrt{E G}}
\end{gathered}
$$

where $\Theta$ is dual angle between $\overrightarrow{R_{12}}$ and $\overrightarrow{R_{22}}$.

Proof. Differentiating (4.10) according to the parameters $v$ and $u$ we have

$$
\frac{\partial \overrightarrow{R_{12}}}{\partial v}=\frac{\overrightarrow{R_{u v}} \sqrt{E}-(\sqrt{E})_{v} \overrightarrow{R_{u}}}{E}
$$

and

$$
\frac{\partial \overrightarrow{R_{22}}}{\partial u}=\frac{\overrightarrow{R_{u v}} \sqrt{G}-(\sqrt{G})_{u} \overrightarrow{R_{v}}}{G}
$$

Then,we get

$$
\begin{gathered}
\overrightarrow{R_{12}} \frac{\partial \overrightarrow{R_{22}}}{\partial u}=\frac{\overrightarrow{R_{u}}}{\sqrt{E}}\left(\frac{\overrightarrow{R_{u v}} \sqrt{G}-(\sqrt{G})_{u} \overrightarrow{R_{v}}}{G}\right)=\frac{(\sqrt{E})_{v}-\cos \Theta(\sqrt{G})_{u}}{\sqrt{G}} \\
\overrightarrow{R_{22}} \frac{\partial \overrightarrow{R_{12}}}{\partial v}=\frac{\overrightarrow{R_{v}}}{\sqrt{G}} \cdot\left(\frac{\overrightarrow{R_{u v}} \sqrt{E}-(\sqrt{E})_{v} \overrightarrow{R_{u}}}{E}\right)=\frac{(\sqrt{G})_{u}-\cos \Theta(\sqrt{E})_{v}}{\sqrt{E}} .
\end{gathered}
$$

On the other hand, considering (4.8) in (4.34) and (4.35) we obtain

$$
\begin{aligned}
& \overrightarrow{R_{12}} \cdot \frac{\partial \overrightarrow{R_{22}}}{\partial S_{1}}=\frac{1}{\sqrt{E}} \overrightarrow{R_{12}} \frac{\partial \overrightarrow{R_{22}}}{\partial u}=\frac{(\sqrt{E})_{v}-\cos \Theta(\sqrt{G})_{u}}{\sqrt{E G}}, \\
& \overrightarrow{R_{22}} \cdot \frac{\partial \overrightarrow{R_{12}}}{\partial S_{2}}=\frac{1}{\sqrt{G}} \overrightarrow{R_{22}} \frac{\partial \overrightarrow{R_{12}}}{\partial v}=\frac{(\sqrt{G})_{u}-\cos \Theta(\sqrt{E})_{v}}{\sqrt{E G}} .
\end{aligned}
$$

Finally, differentiating dual $\operatorname{arcs} S_{1}$ and $S_{2}$, we obtain

$$
\overrightarrow{R_{12}} \cdot \frac{\partial \overrightarrow{R_{22}}}{\partial S_{1}}=-\overrightarrow{R_{22}} \frac{\overrightarrow{R_{12}}}{\partial S_{1}}
$$




$$
\overrightarrow{R_{22}} \cdot \frac{\partial \overrightarrow{R_{12}}}{\partial S_{2}}=-\overrightarrow{R_{12}} \frac{\partial \overrightarrow{R_{22}}}{\partial S_{2}}
$$

Thus,from (4.36) and (4.38),(4.30) is obtained.In a similar way one can obtain (4.31).

Proposition 1. The Blaschke trihedrons $\left\{\overrightarrow{R_{0}}, \overrightarrow{R_{12}}, \overrightarrow{R_{13}}\right\}$ and $\left\{\overrightarrow{R_{0}}, \overrightarrow{R_{22}}, \overrightarrow{R_{23}}\right\}$ of the parameter ruled surfaces of the congruence $\overrightarrow{R(u, v)}$ always coincide such that the dual unit vectors between $\overrightarrow{R_{12}}$ and $\overrightarrow{R_{22}}$ are orthogonal. Moreover, the Blaschke derivative formulae are given by

$$
\begin{gathered}
\frac{d \overrightarrow{R_{12}}}{d S_{2}}=\overrightarrow{B_{2}} \wedge \overrightarrow{R_{12}}, \frac{d \overrightarrow{R_{22}}}{d S_{1}}=\overrightarrow{B_{1}} \wedge \overrightarrow{R_{22}}, \frac{d \overrightarrow{R_{0}}}{d S}=\vec{B} \wedge \overrightarrow{R_{0}}, \\
\frac{d \overrightarrow{R_{12}}}{d S_{1}}=\overrightarrow{B_{1}} \wedge \overrightarrow{R_{12}}, \frac{d \overrightarrow{R_{22}}}{d S_{2}}=\overrightarrow{B_{2}} \wedge \overrightarrow{R_{22}} .
\end{gathered}
$$

Proof. If we take $\Theta=\frac{\pi}{2}$ in (4.22) and (4.23), we have first assertion.

$$
\overrightarrow{R_{13}}=\overrightarrow{R_{22}}, \overrightarrow{R_{23}}=-\overrightarrow{R_{12}}
$$

Differentiating (4.22) according to the dual arc paramater $S_{1}$, we get

$$
\begin{gathered}
\frac{\partial \overrightarrow{R_{13}}}{\partial S_{1}}=\frac{1}{\sin \Theta}\left(-\cos \Theta \frac{\partial \overrightarrow{R_{12}}}{\partial S_{1}}+\frac{\partial \overrightarrow{R_{22}}}{\partial S_{1}}\right), \\
=\frac{1}{\sin \Theta}\left(-\cos \Theta\left(\vec{B}_{1} \wedge \overrightarrow{R_{12}}\right)+\frac{\overrightarrow{R_{22}}}{\partial S_{1}}\right) .
\end{gathered}
$$

Moreover, from (4.41) and (4.22) we know

$$
\frac{\partial \overrightarrow{R_{13}}}{\partial S_{1}}=\vec{B}_{1} \wedge \overrightarrow{R_{13}}=\vec{B}_{1} \wedge\left(\frac { 1 } { \operatorname { s i n } \Theta } \left(-\cos \Theta\left(\vec{B}_{1} \wedge \overrightarrow{R_{12}}\right)+\left(\vec{B}_{1} \wedge \overrightarrow{R_{22}}\right)\right.\right.
$$

Thus, from the second relation of (4.43) and (4.44), we get the second relation of (4.40). Similarly, it can be shown that other relations are satisfied.

Theorem 3. The dual curvatures $Q_{1}$ and $Q_{2}$ of the parameter ruled surfaces $\overrightarrow{R_{11}}$ and $\overrightarrow{R_{21}}$ are given by

$$
\begin{gathered}
Q_{1}=-\frac{1}{\sin \Theta \sqrt{G}}\left((\sqrt{E})_{v}-\cos \Theta(\sqrt{G})_{u}\right), \\
Q_{2}=\frac{1}{\sin \Theta \sqrt{E}}\left((\sqrt{G})_{u}-\cos \Theta(\sqrt{E})_{v}\right) .
\end{gathered}
$$

Proof. From (4.36) and (4.8) we have

$$
\overrightarrow{R_{12}} \cdot \frac{\partial \overrightarrow{R_{22}}}{\partial S_{1}}=\frac{(\sqrt{E})_{v}-\cos \Theta(\sqrt{G})_{u}}{\sqrt{E G}}
$$




$$
\frac{\partial \overrightarrow{R_{22}}}{\partial S_{1}}=\frac{1}{\sqrt{E}} \frac{\partial \overrightarrow{R_{22}}}{\partial u}=\frac{1}{\sqrt{E}} \overrightarrow{B_{1}} \wedge \overrightarrow{R_{22}}
$$

Then, we obtain

$$
\begin{aligned}
& \frac{(\sqrt{E})_{v}-\cos \Theta(\sqrt{G})_{u}}{\sqrt{E G}}=\frac{1}{\sqrt{E}} \overrightarrow{R_{12}} \frac{\partial \overrightarrow{R_{22}}}{\partial u} \\
& =\frac{1}{\sqrt{E}} \overrightarrow{R_{12}}\left(\overrightarrow{B_{1}} \wedge \overrightarrow{R_{22}}\right)=\frac{-\sin \Theta \overrightarrow{R_{0}} \overrightarrow{B_{1}}}{\sqrt{E}}
\end{aligned}
$$

On the other hand, from (4.9), taking dot product $\overrightarrow{B_{1}}$ with $-\sin \Theta \overrightarrow{R_{0}}$ we have

$$
\frac{-\sin \Theta \overrightarrow{R_{0}} \overrightarrow{B_{1}}}{\sqrt{E}}=-\frac{Q_{1} \sin \Theta}{\sqrt{E}} .
$$

Thus, from(4.49) and (4.50) we get $Q_{1}$. Similarly, (4.46) can be obtained.

Theorem 4. Let us consider any ruled surface $\overrightarrow{R_{1}}$ of a line congruence $\overrightarrow{R(u, v)}$ and the dual arc elements of the ruled surfaces $\overrightarrow{R_{1}}, \overrightarrow{R_{11}}$ and $\overrightarrow{R_{22}}$ as $S, S_{1}$ and $S_{2}$, respectively. Let $\overrightarrow{B_{1}}$ and $\overrightarrow{B_{2}}$ be Blaschke vectors of $\overrightarrow{R_{11}}$ and $\overrightarrow{R_{21}}$. We also suppose that the dual angle between the line $\overrightarrow{R_{2}}$ and $\overrightarrow{R_{12}}$ is $\Phi$, then we have the following relations

$$
\vec{C}=\frac{1}{P_{1}} \frac{\sin (\Theta-\Phi)}{\sin \Theta} \vec{B}_{1}+\frac{1}{P_{2}} \frac{\sin \Phi}{\sin \Theta} \vec{B}_{2}
$$

and

$$
\frac{d \overrightarrow{R_{12}}}{d S}=\vec{C} \wedge \overrightarrow{R_{12}}, \frac{d \overrightarrow{R_{22}}}{d S}=\vec{C} \wedge \overrightarrow{R_{22}}, \frac{d \overrightarrow{R_{0}}}{d S}=\vec{C} \wedge \overrightarrow{R_{0}}
$$

Proof. We know that the dual vector $\overrightarrow{R_{12}}$ is the function of $S_{1}$ and $S_{2}$. Hence we have

$$
\frac{d \overrightarrow{R_{12}}}{d S}=\frac{\partial \overrightarrow{R_{12}}}{\partial S_{1}} \frac{d S_{1}}{d S}+\frac{\partial \overrightarrow{R_{12}}}{d S_{2}} \frac{d S_{2}}{d S}
$$

From the equalities (4.19), (4.20), (4.40) and (4.41) we obtain

$$
\begin{gathered}
\frac{d R_{12}}{d S}=\frac{\sin (\Theta-\Phi)}{\sin \Theta} \frac{1}{\sqrt{E}}\left(\overrightarrow{B_{1}} \wedge \overrightarrow{R_{12}}\right)+\frac{\sin \Phi}{\sin \Theta} \frac{1}{\sqrt{E}}\left(\overrightarrow{B_{2}} \wedge \overrightarrow{R_{12}}\right) \\
=\left(\frac{\sin (\Theta-\Phi)}{\sin \Theta} \frac{1}{P_{1}} \overrightarrow{B_{1}}+\frac{\sin \Phi}{\sin \Theta} \frac{1}{P_{2}} \overrightarrow{B_{2}}\right) \wedge \overrightarrow{R_{12}} \\
=\vec{C} \wedge \overrightarrow{R_{12}} .
\end{gathered}
$$

Thus, we have the first relation of (4.52). Other assertion can be obtained. 
Theorem 5. Let us consider the ruled surfaces $\overrightarrow{R_{1}}, \overrightarrow{R_{11}}$ and $\overrightarrow{R_{22}}$ which have common line $\overrightarrow{R_{0}}$ on the line congruence $\overrightarrow{R(u, v)}$. Let $\overrightarrow{B_{1}}$ and $\overrightarrow{B_{2}}$ be Blaschke vectors of $\overrightarrow{R_{11}}$ and $\overrightarrow{R_{21}}$. We obtain following equality among the Blaschke vectors

$$
\vec{B}=P\left(\frac{1}{P_{1}} \frac{\sin (\Theta-\Phi)}{\sin \Theta} \vec{B}_{1}+\frac{1}{P_{2}} \frac{\sin \Phi}{\sin \Theta} \vec{B}_{2}+\frac{d \Phi}{d S} \vec{R}_{0}\right)
$$

Proof. From (4.17), we have

$$
\overrightarrow{R_{2}}=\frac{\sin (\Theta-\Phi)}{\sin \Theta} \overrightarrow{R_{12}}+\frac{\sin \Phi}{\sin \Theta} \overrightarrow{R_{22}}
$$

Then, by taking derivative with respect to dual arc $S$, from equation (4.17), we obtain

$$
\begin{gathered}
\frac{d \overrightarrow{R_{2}}}{d S}=\frac{d \overrightarrow{R_{12}}}{d S} \frac{\sin (\Theta-\Phi)}{\sin \Theta}+\frac{d \overrightarrow{R_{22}}}{d S} \frac{\sin \Phi}{\sin \Theta}- \\
\frac{\cos (\Theta-\Phi)}{\sin \Theta} \frac{d \Phi}{d S} \overrightarrow{R_{12}}+\frac{\cos \Phi}{\sin \Theta} \frac{d \Phi}{d S} \overrightarrow{R_{22}}
\end{gathered}
$$

On the other hand, considering the Blaschke trihedrons $\left\{\overrightarrow{R_{0}}, \overrightarrow{R_{12}}, \overrightarrow{R_{13}}\right\}$ and $\left\{\overrightarrow{R_{0}}, \overrightarrow{R_{22}}, \overrightarrow{R_{23}}\right\}$, we write

$$
\begin{gathered}
\overrightarrow{R_{13}}=\overrightarrow{R_{12}} \wedge \overrightarrow{R_{0}}=\overrightarrow{R_{12}} \wedge\left(\frac{\overrightarrow{R_{12}} \wedge \overrightarrow{R_{22}}}{\sin \Theta}\right) \\
=\frac{\left(<\overrightarrow{R_{12}}, \overrightarrow{R_{22}}>\overrightarrow{R_{12}}-<\overrightarrow{R_{12}}, \overrightarrow{R_{12}}>\overrightarrow{R_{22}}\right)}{\sin \Theta} \\
=\frac{1}{\sin \Theta}\left(\cos \Theta \overrightarrow{R_{12}}-\overrightarrow{R_{22}}\right)
\end{gathered}
$$

and

$$
\begin{gathered}
\overrightarrow{R_{23}}=\overrightarrow{R_{22}} \wedge \overrightarrow{R_{0}}=\overrightarrow{R_{22}} \wedge\left(\frac{\overrightarrow{R_{12}} \wedge \overrightarrow{R_{22}}}{\sin \Theta}\right) \\
=\frac{<\overrightarrow{R_{22}}, \overrightarrow{R_{22}}>\overrightarrow{R_{12}}-<\overrightarrow{R_{22}}, \overrightarrow{R_{12}}>\overrightarrow{R_{22}}}{\sin \Theta} \\
=\frac{1}{\sin \Theta}\left(\overrightarrow{R_{12}}-\cos \Theta \overrightarrow{R_{22}}\right) .
\end{gathered}
$$

And then, substituting the equations (4.57) and (4.58) in $-\overrightarrow{R_{12}}=\overrightarrow{R_{0}} \wedge \overrightarrow{R_{13}}$ and $-\overrightarrow{R_{22}}=\overrightarrow{R_{0}} \wedge \overrightarrow{R_{23}}$ we have (4.55) as follows

$$
\begin{gathered}
\frac{d \overrightarrow{R_{2}}}{d S}=\frac{d \overrightarrow{R_{12}}}{d S} \frac{\sin (\Theta-\Phi)}{\sin \Theta}+\frac{d \overrightarrow{R_{22}}}{d S} \frac{\sin \Phi}{\sin \Theta} \\
+\left[-\overrightarrow{R_{0}} \wedge \frac{\left(\cos \Theta \overrightarrow{R_{12}}-\overrightarrow{R_{22}}\right)}{\sin \Theta} \frac{\cos (\Theta-\Phi)}{\sin \Theta}+\overrightarrow{R_{0}} \wedge \frac{\left(\overrightarrow{R_{12}}-\cos \Theta \overrightarrow{R_{22}}\right)}{\sin \Theta} \frac{\cos \Phi}{\sin \Theta}\right] \frac{d \Phi}{d S} .
\end{gathered}
$$


According to theorem 4, we have

$$
\begin{gathered}
\frac{d \overrightarrow{R_{12}}}{d S}=\vec{C} \wedge \overrightarrow{R_{12}}, \frac{d \overrightarrow{R_{22}}}{d S}=\vec{C} \wedge \overrightarrow{R_{22}}, \\
\vec{C}=\frac{1}{P_{1}} \frac{\sin (\Theta-\Phi)}{\sin \Theta} \vec{B}_{1}+\frac{1}{P_{2}} \frac{\sin \Phi}{\sin \Theta} \vec{B}_{2} .
\end{gathered}
$$

By using the dual trigonometric expression, we find

$$
\begin{gathered}
\frac{d \overrightarrow{R_{2}}}{d S}=\frac{\sin (\Theta-\Phi)}{\sin \Theta} \vec{C} \wedge \overrightarrow{R_{12}}+\frac{\sin \Phi}{\sin \Theta} \vec{C} \wedge \overrightarrow{R_{22}}+ \\
\overrightarrow{R_{0}} \wedge\left[\frac{\sin (\Theta-\Phi)}{\sin \Theta} \overrightarrow{R_{12}}+\frac{\sin \Phi}{\sin \Theta} \overrightarrow{R_{22}}\right] \frac{d \Phi}{d S} \\
=\left(\vec{C}+\overrightarrow{R_{0}} \frac{d \Phi}{d S}\right) \wedge \overrightarrow{R_{2}} .
\end{gathered}
$$

Since we have

$$
\vec{M}=\vec{C}+\overrightarrow{R_{0}} \frac{d \Phi}{d S}
$$

and

$$
\vec{C}=\vec{M}-\overrightarrow{R_{0}} \frac{d \Phi}{d S}
$$

Thus, (4.62) is written by

$$
\frac{d \overrightarrow{R_{2}}}{d S}=\vec{M} \wedge \overrightarrow{R_{2}}
$$

From the last equation of (4.52) we have

$$
\begin{gathered}
\frac{d \overrightarrow{R_{0}}}{d S}=\vec{C} \wedge \overrightarrow{R_{0}} \\
=\left(\vec{M}-\overrightarrow{R_{0}} \frac{d \Theta}{d S}\right) \wedge \overrightarrow{R_{0}} \\
=\left(\vec{M} \wedge \overrightarrow{R_{0}}\right)
\end{gathered}
$$

and

$$
\frac{d \overrightarrow{R_{0}}}{d S}=\vec{M} \wedge \overrightarrow{R_{0}}
$$


By using the Blaschke vector of $\frac{d \overrightarrow{R_{2}}}{d S}$ and (4.65) we obtain

$$
\begin{gathered}
\vec{M} \wedge \overrightarrow{R_{2}}-\vec{B} \wedge \overrightarrow{R_{2}}=0 \\
(\vec{M}-\vec{B}) \wedge \overrightarrow{R_{2}}=0 \\
\vec{M}-\vec{B}=\Lambda \overrightarrow{R_{2}} ; \Lambda \varepsilon D
\end{gathered}
$$

and

$$
\begin{gathered}
\vec{M} \wedge \overrightarrow{R_{2}}-\vec{B} \wedge \overrightarrow{R_{0}}=0 \\
(\vec{M}-\vec{B}) \wedge \overrightarrow{R_{0}}=0 \\
\vec{M}-\vec{B}=\Omega \overrightarrow{R_{0}}, \Omega \varepsilon D .
\end{gathered}
$$

From (4.67) and (4.68) we have

$$
\Lambda \overrightarrow{R_{2}}=\Omega \overrightarrow{R_{0}}
$$

$$
\Lambda=\Omega=0
$$

Consequently, we have obtained

$$
\vec{M}=\vec{B}
$$

and hence we conclude

$$
\vec{B}=\vec{C}+\overrightarrow{R_{0}} \frac{d \Theta}{d S}
$$

Corollary 3. Let $\vec{R}$ and $\overrightarrow{R_{11}}, \overrightarrow{R_{21}}$ be a ruled surface and parameter ruled surface of the line congruence $R(u, v)$, respectively.Then, we have

$$
\Sigma=\Sigma_{1} \frac{\sin (\Theta-\Phi)}{\sin \Theta}+\Sigma_{2} \frac{\sinh \Phi}{\sin \Theta}+\frac{d \Phi}{d S}
$$

where $\Theta$ is dual angle between $\overrightarrow{R_{12}}$ and $\overrightarrow{R_{22}}, \Phi$ is dual angle between $\overrightarrow{R_{2}}$ and $\overrightarrow{R_{12}}, \Sigma$ is dual spherical curvature of the ruled surface $\overrightarrow{R_{1}}, \Sigma_{1}$ is dual spherical curvature of the ruled surface $\overrightarrow{R_{11}}, \Sigma_{2}$ is dual spherical curvature of the ruled surface $\overrightarrow{R_{22}}$.

Proof. If we substitute the Blaschke vectors $\vec{B}, \overrightarrow{B_{1}}$ and $\overrightarrow{B_{2}}$ in (4.54) and then taking dot product of both sides by $\overrightarrow{R_{0}}$, we have

$$
Q=P\left(\frac{Q_{1}}{P_{1}} \frac{\sin (\Theta-\Phi)}{\sin \Theta}+\frac{Q_{2}}{P_{2}} \frac{\sin \Phi}{\sin \Theta}+\frac{d \Phi}{d S}\right)
$$


Thus, considering (3.3) we get assertion.

Theorem 6.(Mannheim's Formula) The following relation

$$
\left.P=P_{1} \frac{\sin (\Theta-\Phi)}{\sin \Theta}+P_{2} \frac{\sinh \Phi}{\sin \Theta}\right]
$$

is satisfied among the dual curvatures of ruled surfaces $\overrightarrow{R_{1}}, \overrightarrow{R_{11}}$ and $\overrightarrow{R_{21}}$ of the line congruence $\overrightarrow{R(u, v)}$.

Proof.Substituting the Blaschke vectors $\vec{B}, \overrightarrow{B_{1}}$ and $\overrightarrow{B_{2}}$ in (4.54) and then taking dot product both of sides by $\overrightarrow{R_{3}}$, desired equation is obtained.

Theorem 7. (Liouville's Formula) There are following relation among the dual torsions of the ruled surfaces $\overrightarrow{R_{1}}, \overrightarrow{R_{11}}$ and $\overrightarrow{R_{21}}$ of the spacelike line congruence $\overrightarrow{R(u, v)}$ as

$$
Q=Q_{1} \frac{\sin (\Theta-\Phi)}{\sin \Theta}+Q_{2} \frac{\sin \Phi}{\sin \Theta}+\frac{d \Phi}{d S}
$$

Proof. If we substitute the Blaschke vectors $\vec{B}, \overrightarrow{B_{1}}$ and $\overrightarrow{B_{2}}$ in (4.54) and then taking dot product both of sides by $\overrightarrow{R_{0}}$, desired equation is obtained.

\section{References}

[1] Ravani, B., Ku Ts.,Bertrand offsets of ruled and developable surface, Computer Aided Geometric Design, Elsevier, 23(2), 145-152, 1991.

[2] Caliskan, A., On the Studying of a line Congruence by Choosing Parameter Ruled Surfaces as Principal Ruled Surface. Journal of Faculty Science of Ege University Series A, No. (1),1987.

[3] Caliskan, A., The Relation Among Blaschke Vectors of Ruled Surfaces on a Line Congruence And Its Consequance, Commun. Fac. Sci. Univ. Ank. Series An, Number (1-2), pp. 77-86,1989.

[4] W. Blaschke,Vorlesungen uber Differentialgeometrie und geometrische Grundlagen von Einsteins Relativit atstheorie, Dover Publications, New York, 1945.

[5] Kummer. E., Aber die algebraischen Strahlensysteme. insbesondere uber die derersten undzweiten Ordnung. Abh. K. Preuss. Akad. Wiss. Berlin (1866), 1-120, also in E. E. Kummer. Collected Papers. Springer Verlag, 1975.

[6] Gugenheimer. H. W., Differential Geometry, Graw-Hill, New York, 1956.

[7] Karger, A. , Novak, J., Space Kinematics and Line Groups. Gordon and Breach Science Publishers. New York, 1985.

[8] Veldkamp, G. R., On the use of dual numbers, vectors and matrices in instantaneous spatial kinematics, Mech. Mach. Theory 11 (1976), 141-156.

[9] Clifford, W. K., Preliminary sketch of bi-quaternions. Proc. London Math. Soc. 4 (64,65), (1873). 361-395.

[10] Muller, H. R., Kinematik Dersleri. Ankara University Press, 1963. 